\title{
Call for Papers: Special Issue of Earth, Planets and Space (EPS)
}

We now invite papers for a special issue of EPS entitled "Itokawa-Hayabusa and beyond." This special issue will be based on, but not limited to, the works related to observations by instruments on board the Hayabusa spacecraft. Papers presented in the 2nd Hayabusa symposium held on July 12-14 in Tokyo are encouraged to be submitted to this special issue. Papers based on ground-based observations and experimental works addressing the themes of the special issue are also welcome. Suitable subjects include the origin and evolution of (near-Earth) asteroids and their connection with meteorites.

Contributors to this special issue should submit their papers directly to the EPS editorial office either by post or by e-mail (eps@terrapub.co.jp), following the instructions for regular EPS submission. For details, please visit the following web page:

http://www.terrapub.co.jp/journals/EPS and click on "Information for Contributors."

Please state "For Special Issue for Hayabusa" clearly in the cover letter when submitting. We encourage electronic submissions. The submitted papers will be peer reviewed as are all EPS regular papers. After papers are accepted, the authors will receive instructions for the final manuscript from the editorial office.

The deadline for manuscript submissions for this special issue is August 31, 2006. We expect the special issue to be published in January 2007.

For more information on this special issue, please contact the corresponding editor (Sho Sasaki: sho@miz.nao.ac.jp) while questions on manuscript preparation should be addressed to the EPS editorial office.

Guest Editors: Sho Sasaki, sho@miz.nao.ac.jp

Donald K. Yeomans, Donald.K.Yeomans@jpl.nasa.gov

Akiko M. Nakamura, amnakamu@kobe-u.ac.jp 